\title{
Detection of monocyte-derived microparticles in patients with Type II diabetes mellitus
}

\author{
S. Omoto ${ }^{1}$, S. Nomura ${ }^{2}$, A.Shouzu ${ }^{1}$, M.Nishikawa ${ }^{1}$, S. Fukuhara ${ }^{2}$, T. Iwasaka ${ }^{1}$ \\ ${ }^{1}$ Second Department of Internal Medicine, Kansai Medical University, Osaka, Japan \\ ${ }^{2}$ First Department of Internal Medicine, Kansai Medical University, Osaka, Japan
}

\begin{abstract}
Aims/hypothesis. The role of plasma monocyte-derived microparticles (MDMPs) and platelet-activation markers (platelet-derived microparticle [PDMP], platelet-bound CD62P [plt-CD62P], and platelet-bound CD63 [plt-CD63]) in diabetic vascular complications is not clear. We measured and compared plasma concentrations of MDMPs and the platelet-activation markers to investigate their possible contribution to diabetic vascular complications.

Methods. Activated platelets and microparticles (PDMP and MDMP) were analysed by flow cytometry. Concentrations of serum sE-selectin were measured with enzyme-linked immunosorbent assay.

Results. The concentration of MDMPs in diabetic patients was higher than in normal subjects. We found no differences in the binding of anti-GPIIb/IIIa and anti-GPIb monoclonal antibodies between groups. There were differences, however, in the concentrations of PDMPs, plt-CD62P, and plt-CD63 between Type II (non-insulin-dependent) diabetes mellitus patients and control subjects (PDMPs: $585 \pm 25$ vs $263 \pm 9, \quad p<0.01$; plt-CD62P: $28.1 \% \pm 1.4 \%$ vs $9.4 \% \pm 0.6 \%, p<0.001$; plt-CD $63: 28.1 \% \pm 1.4 \%$ vs $8.6 \% \pm 0.5 \%, p<0.001)$. Amounts of MDMPs corre-
\end{abstract}

lated positively with these platelet activation markers, and the relation between PDMP and MDMP was the most significant. The concentration of MDMP in patients who had diabetes complicated with nephropathy, retinopathy, or neuropathy was higher than in those without diabetes-related complications. The increase in MDMP was particularly significant in patients with nephropathy. Concentrations of sE-selectin were higher in Type II diabetes patients than in control subjects, and correlated with MDMP, PDMP, plt-CD62P, and plt-CD63 levels in nephropathy patients.

Conclusion/interpretation. In Type II diabetes patients, we detected increased activation of monocytes, which could have been stimulated by activated platelets and PDMPs. Because the activation of monocytes is associated with vascular endothelial damage, high concentrations of MDMPs could indicate vascular complications in diabetes patients, especially those who have diabetes-related nephropathy. [Diabetologia (2002) 45:550-555]

Keywords Monocyte-derived microparticle, Type II diabetes mellitus, soluble E-selectin, platelet-derived microparticle, activated platelets.
Received: 21 May 2001 and in revised form: 13 November 2001

Corresponding author: S. Nomura, First Department of Internal Medicine, Kansai Medical University, 10-15 Fumizonocho, Moriguchi Osaka 570-8507, Japan, e-mail: shosakun@mbp.sphere.ne.jp

Abbreviations: MDMP, Monocyte-derived microparticles; PDMP, plasma-derived microparticles; plt-CD62P, plateletbound CD62P; plt-CD63, platelet-bound CD63; PRP, platelet-rich plasma; FITC-AnnV, FITC-labelled annexin V; sE-selectin, soluble E-selectin
Patients with diabetes mellitus develop hypercoagulability, platelet hyperaggregability and premature atherosclerosis [1-4]. Atherosclerosis is the major cause of death in patients with diabetes mellitus, leading to a high mortality rate in all forms of the disease [5]. Classical risk factors, including hyperlipidaemia, hypertension, and obesity, do not completely account for the increased incidence of atherosclerosis associated with diabetes [6]. Recent reports suggest that increased expression of endothelial adhesion 
molecules plays a role in the acceleration of diabetic macro-angiopathy [7-11]. In addition, there have been some reports that activated platelets and platelet-derived microparticles (PDMPs) are higher in Type II diabetes patients [12-15].

Monocytes and macrophages could play important roles in the development of atherosclerosis [16-19]. The first step in monocyte infiltration into the subendothelial space is adhesion of circulating monocytes to the endothelium. Macrophages predominate in the lipid core of atherosclerotic plaque, and they have been shown to express tissue factor [20-22]. Monocytes can synthesize procoagulants, mostly to the tissue factor [23,24]. Monocyte vesiculation could disseminate membrane-associated procoagulant activities and adhesion molecules following stimulation by lipopolysaccharide [25]. This ability of endotoxinstimulated monocytes to release heterogeneous monocyte-derived microparticles (MDMPs) could be an important mechanism regulating vascular cell effector functions [26]. The tissue factor-exposing MDMPs promote prothrombinase complex assembly, thereby facilitating intravascular generation of thrombin and enhanced procoagulant activity [27]. Investigators recently reported that MDMP retained tissue factor activity in atherosclerotic plaques [28].

Cell adhesion molecules are thought to be involved in the development of vascular complications in diabetes mellitus and show increased expression levels in patients with both Type I and Type II diabetes [10]. E-selectin is a cell adhesion molecule that contributes to monocyte adhesion to endothelium [29] and is expressed by endothelial cells at the site of atheroscrelotic lesions [30]. Serum concentrations of soluble E-selectin (sE-selectin) are higher in patients with diabetes mellitus [9-11].

Procoagulant activity is also found in markers of platelet activation, such as platelet-bound CD62P (plt-CD62P) and PDMPs [31,32]. Thus, monocytes, platelets, and E-selectin could be very important for the development of atherosclerosis in diabetes patients. We measured and compared the concentrations of plasma MDMP and platelet activation markers [plasma PDMP, CD62P binding to platelets; pltCD62P, CD63 binding to platelets; plt-CD63], to develop a better understanding of their potential contribution to diabetic vascular complications.

\section{Subjects and methods}

Patients. The study group included 50 healthy control subjects and 73 Type II diabetic patients. All gave informed consent according to the Declaration of Helsinki. Table 1 shows the characteristics of patients and control subjects. Diabetic complications included symptomatic neuropathy treated medically (23 cases, $31.5 \%$ ), retinopathy (34 cases, $46.6 \%$ ), and nephropathy (29 cases, $39.7 \%$ ) (Table 2). Diabetic nephropathy was diagnosed by the presence of macroalbuminurea (>300 mg/
Table 1. Clinical characteristics of patients and healthy control subjects

\begin{tabular}{lcc}
\hline & $\begin{array}{c}\text { Control subjects } \\
(n=50)\end{array}$ & $\begin{array}{c}\text { Type II diabetes } \\
\text { patients }(n=73)\end{array}$ \\
\hline Men/women $(n)$ & $29 / 21$ & $30 / 43$ \\
Age $($ years $)$ & $51 \pm 7$ & $54 \pm 6$ \\
$\mathrm{BMI}\left(\mathrm{kg} / \mathrm{m}^{2}\right)$ & $22.4 \pm 1.1$ & $22.9 \pm 0.4$ \\
$\mathrm{WBC}(/ \mu \mathrm{l})$ & $4720 \pm 514$ & $5330 \pm 693$ \\
Platelet count $\left(\times 10^{4} / \mu \mathrm{l}\right)$ & $25.4 \pm 4.2$ & $26.1 \pm 5.8$ \\
HbA $_{1 \mathrm{c}}(\%)$ & $4.9 \pm 0.5$ & $7.9 \pm 0.6^{*}$ \\
Total cholesterol $(\mathrm{mg} / \mathrm{dl})$ & $213 \pm 41$ & $231 \pm 39$ \\
Triglycerides $(\mathrm{mg} / \mathrm{dl})$ & $146 \pm 35$ & $164 \pm 41^{* *}$ \\
\hline
\end{tabular}

Data are shown as means \pm SEM

$* p<0.001$

$* * p<0.05$

Table 2. Relation between PMP/platelet activation markers in control subjects and Type II diabetes patients

\begin{tabular}{lcl}
\hline & $\begin{array}{l}\text { Control subjects } \\
(n=50)\end{array}$ & $\begin{array}{l}\text { Type II diabetes } \\
\text { patients }(n=73)\end{array}$ \\
\hline plt-gpIIb/IIIa (\%) & $95.3 \pm 3.1$ & $95.2 \pm 2.9$ \\
plt-gpIb (\%) & $92.9 \pm 3.6$ & $93.1 \pm 3.8$ \\
plt-CD62P (\%) & $9.4 \pm 0.6$ & $28.1 \pm 1.4^{*}$ \\
plt-CD63 (\%) & $8.6 \pm 0.5$ & $28.1 \pm 1.4^{*}$ \\
PDMP $\left(/ 10^{4}\right.$ plt) & $263 \pm 9$ & $585 \pm 25^{*}$ \\
\hline
\end{tabular}

Plt, platelets; Values are shown as means \pm SEM

$* p<0.001$

day) in more than three consecutive urine samples. Serum creatinine was lower than $2.0 \mathrm{mg} / \mathrm{dl}$ in all patients. Five patients diagnosed with nephropathy had macroangiopathy. Evaluation of neuropathy was done by neurological examination and revealed reduced periosteal reflex and vibratory sensation, and what was diagnosed for patients with neurological symptoms. No patients had inflammatory markers such as CRP and increased leukocyte counts. Anti-thrombotic agents, including aspirin, were not administered but ACE inhibitor was given to 18 patients.

Flow cytometry of activated platelets and microparticles. Blood samples were collected into tubes containing $3.8 \%$ sodium citrate $(9: 1 \mathrm{vol} / \mathrm{vol})$. Platelet-rich plasma (PRP) was prepared by centrifugation at $200 \mathrm{~g}$ for $10 \mathrm{~min}$ at room temperature. Washed platelets were prepared by centrifuging PRP at $1400 \mathrm{~g}$ for $10 \mathrm{~min}$ at room temperature. The resultant pellet was washed twice with washing buffer $\left(9 \mathrm{mmol} / \mathrm{l} \mathrm{Na}_{2}\right.$ EDTA, $140 \mathrm{mmol} / \mathrm{l} \mathrm{NaCl}$, and $\left.26 \mathrm{mmol} / \mathrm{N} \mathrm{Na}_{2} \mathrm{HPO}_{4}, \mathrm{pH} 7.2\right)$ and resuspended in HEPES-Tyrode's buffer (129 mmol/l $\mathrm{NaCl}$, $8.9 \mathrm{mmol} / \mathrm{l} \mathrm{NaHCO}, 0.8 \mathrm{mmol} / \mathrm{KH}_{2} \mathrm{PO}_{4}, 0.8 \mathrm{mmol} / 1 \mathrm{MgCl}_{2}$, $5.6 \mathrm{mmol} / \mathrm{l}$ glucose, and $10 \mathrm{mmol} / \mathrm{l} \mathrm{HEPES}, \mathrm{pH} 7.4)$. An equal volume of $2 \%$ paraformaldehyde was added to the washed platelets, and they were incubated for $15 \mathrm{~min}$ at room temperature. Platelets were then washed twice, resuspended in stock solution (9 mmol/l Na $\mathrm{EDTA}_{2} 26.4 \mathrm{mmol} / \mathrm{l} \mathrm{Na} \mathrm{HPO}_{4} 2 \mathrm{H}_{2} \mathrm{O}$, $140 \mathrm{mmol} / \mathrm{l} \mathrm{NaCl}, 0.1 \% \mathrm{NaN}_{3}$, and $2 \%$ fetal bovine serum, $\mathrm{pH}$ 7.2), and stored at $4{ }^{\circ} \mathrm{C}$ until analysis.

PDMPs were detected with a modified version of a previously reported method $[12,33,34]$. Ten microlitres of platelets $\left(3 \times 10^{8} / \mathrm{ml}\right)$ added to $100 \mu \mathrm{l}$ of HEPES-Tyrode's buffer containing $5 \mathrm{nmol} / 1$ EGTA. Both intact and aggregated platelets were removed by centrifugation at $1000 \mathrm{~g}$ for $15 \mathrm{~min}$ to yield a 


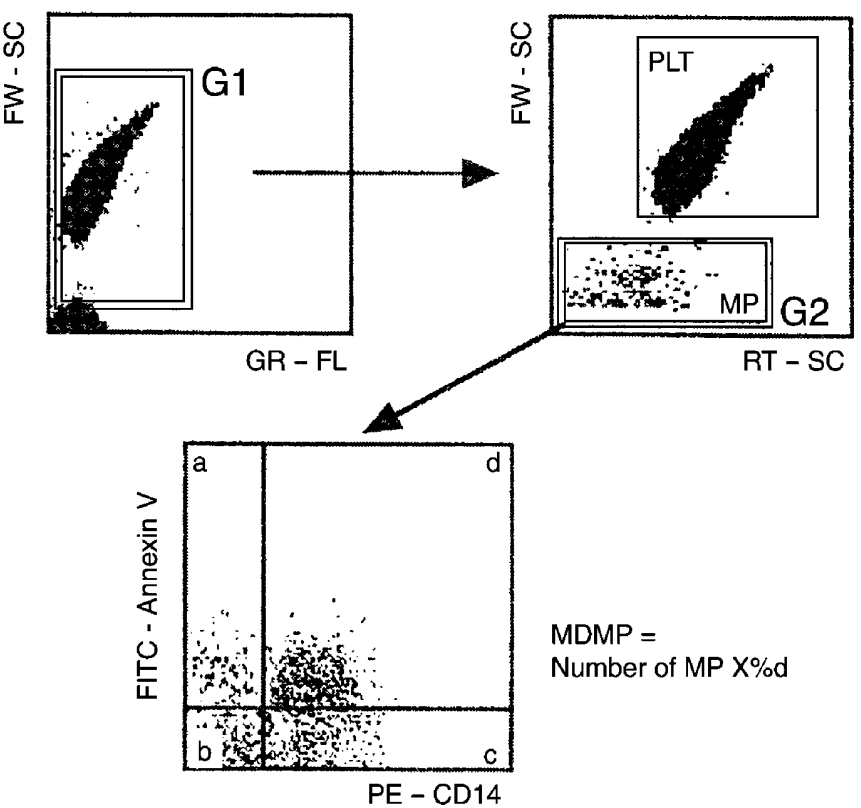

Fig. 1. Assessment of MDMP. Washed intact platelets was added to the plasma and the mixture was incubated with FITC-labelled Annexin V and PE-labelled CD14 for $30 \mathrm{~min}$ in the dark at room temperature. Samples were diluted 1:10 with HEPES-Tyrode's and analysed with Flow cytometry. Flow cytometry was set to detect only particles bound to FITC-labelled Annexin V and PE-labelled CD14

supernatant containing microparticles only. Ten microlitres of washed intact platelets $\left(3 \times 10^{8} / \mathrm{ml}\right)$ was then added to the supernatant and the mixture was incubated with KMP-9 [35] (a FITC-labelled monoclonal antibody against platelet GPIX) for $30 \mathrm{~min}$ in the dark at room temperature. After incubation, samples were diluted 1:10 with HEPES-Tyrode's buffer containing $5 \mathrm{nmol} / \mathrm{l}$ EGTA and analysed with an Ortho Cytoron Absolute Analyzer (Ortho Diagnostic Systems, Tokyo, Japan). Only cells and particles positive for GPIX were gated to distinguish platelets and PDMPs from electronic noise. To differentiate between platelets and PDMPs, the lower limit of the platelet gate was set at the left limit of the forward-scatter profile of resting platelets. Then 10000 FITC-positive particles in the PDMP gate were to determine the number of PDMPs released for each 10000 platelets. As an index of platelet activation, CD62P expression was quantified by immunostaining with anti-CD62P monoclonal antibody (CLB-thromb/6, Immunotech, Marseille, France). Platelet expression of GPIIb/ IIIa and of GPIb was analysed with anti-GPIIb/IIIa (NNKY1-32)[33,36] and anti-GPIb (NNKY5-5)[37] monoclonal antibodies, respectively.

Assessment of MDMP. MDMP was detected by a previously reported method with some modifications [38]. Ten microlitres of washed intact platelets $\left(3 \times 10^{8} / \mathrm{ml}\right)$ was added to the plasma, and the mixture was incubated with FITC-labelled Annexin V (FITC-Ann V) and phycoerythrin (PE)-labelled CD14 (PE-CD14) for $30 \mathrm{~min}$ in the dark at room temperature. Samples were diluted 1:10 with HEPES-Tyrode's buffer containing $5 \mathrm{nmol} / \mathrm{l}$ EGTA and analysed with an Ortho Cytoron Absolute Analyzer (Ortho Diagnostics). Flow cytometry was set to detect only particles bound to FITC-Ann V and PE-CD14 (Fig. 1).

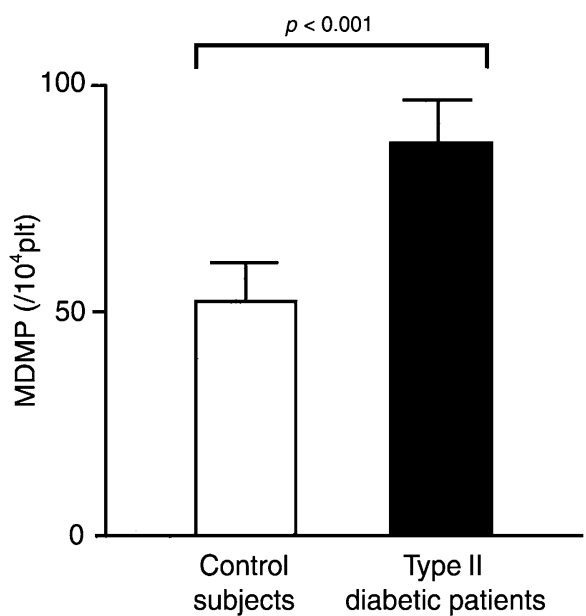

Fig. 2. The results of MDMP in normal subjects and Type II diabetic patients. The serum level of MDMP in diabetic patients was higher than that in normal subjects

Measurement of sE-selectin. Blood samples from patients and healthy control subjects were collected into tubes with sodium citrate or tubes without anticoagulant. Blood was allowed to clot at room temperature for a minimum of $1 \mathrm{~h}$ serum or citrated plasma was isolated by centrifugation for $20 \mathrm{~min}$ at $1000 \mathrm{~g}$ at $4{ }^{\circ} \mathrm{C}$ and then stored at $-30^{\circ} \mathrm{C}$ until analysis. As positive controls, in each assay we used the recombinant products and standard solutions provided with the commercial kits. Concentrations of serum sE-selectin were measured with a monoclonal antibody-based ELISA kit from R \& D Systems according to the manufacturer's instructions.

Statistics. Statistical analysis was performed with the paired or unpaired $t$-test, and a $p$ value of less than 0.05 was considered to be statistically significant.

\section{Results}

We compared the results of MDMP in normal subjects with those in patients with Type II diabetes (Fig. 2). The concentration of MDMP in diabetic patients was higher $(p<0.001)$ than that in normal subjects.

When concentrations of PDMPs and platelet activation markers were compared between the control and Type II diabetes groups, no between-group differences were detected in binding of anti-GPIIb/IIIa and anti-GPIb monoclonal antibodies (Table 2). Concentrations of PDMPs, plt-CD62P, and plt-CD63, however, were higher $(p<0.001$ for each) in Type II diabetes patients than in control subjects (Table 2 ).

MDMP correlated positively with plt-CD62P, pltCD63, and PDMP with its relation to PDMP being particularly significant (Fig.3). However, $\mathrm{HbA}_{1 \mathrm{c}}$, an indicator of glycaemic control did not correlate with MDMP, PDMP, plt-CD62P, or plt-CD63 concentrations (data not shown).

The number of MDMPs is patients who have diabetes together with complications such as nephropa- 


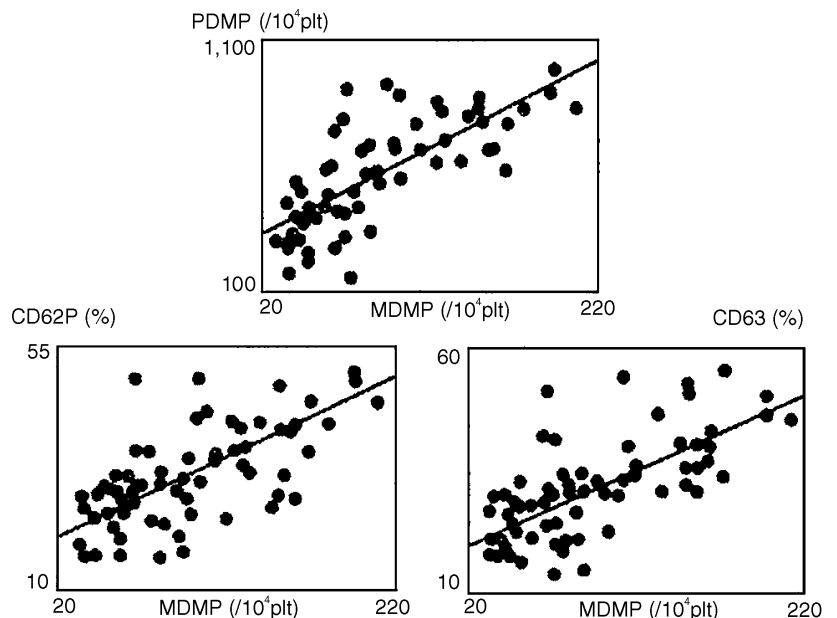

Fig. 3. The correlation between MDMP and platelet activation markers (plt-CD62P, plt-CD63, and PDMP). MDMP correlated positively with the platelet activation markers, with the PDMP relation being particularly significant

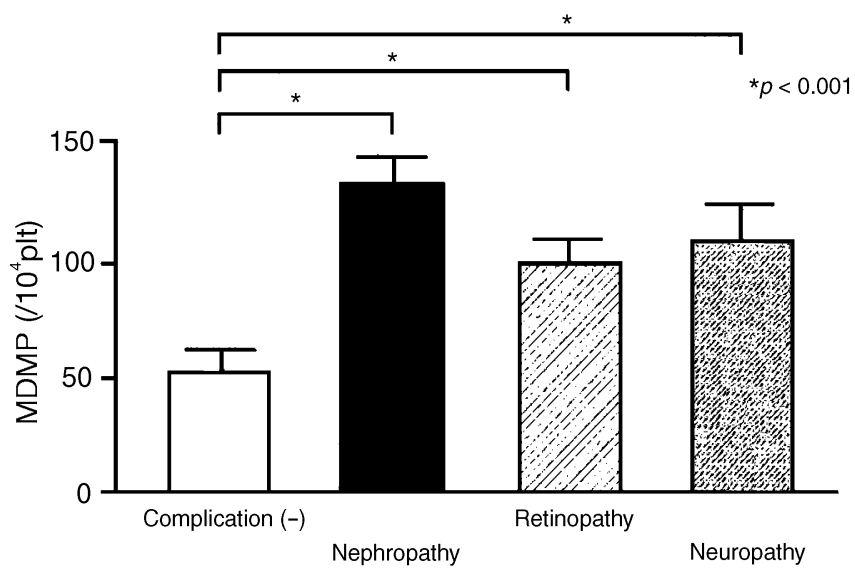

Fig. 4. The concentration of MDMP in patients with diabetes complicated with nephropathy, retinopathy, or neuropathy was higher than that in those without complications. The increase of MDMP was most significant in patients with nephropathy

thy, retinopathy, or neuropathy were higher than that in those without complications with nephropathy being the highest (Fig. 4).

Concentrations of sE-selectin were higher in Type II diabetes patients than in control subjects (Fig.5). In addition, sE-selectin concentrations correlated with MDMP, PDMP, plt-CD62P, and plt-CD63 in nephropathy patients (Table 3 ). In this study, ACEinhibitor was given to 18 patients. However, values of platelet activation markers, MDMP, and sE-selectin did not differ significantly between the administration group and the non-administration group.

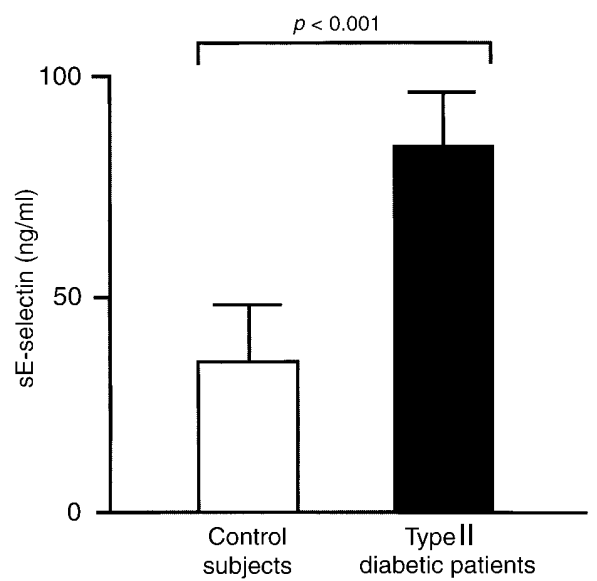

Fig.5. Concentrations of sE-selectin in Type II diabetic patients $(n=73)$ and control subjects $(n=50)$. Data are means \pm SEM

\section{Discussion}

This study shows that concentrations of plt-CD62P, PDMP, and MDMP are higher in patients with diabetes, and that these increases are the highest in diabetes complicated by nephropathy.

Diabetic macro-angiopathy originates from atherosclerosis, and it is believed that the coagulation or fibrinolysis system or both cause it. In fibrinolytic abnormalities, plasminogen activator inhibitor 1 (PAI-1) plays a leading role. Low plasma fibrinolytic activity in association with increased plasma PAI-1 levels has been linked to an increased risk of atherosclerosis in patients with Type II diabetes [39-41]. Hyperactive platelets could lead to capillary microembolization because of the formation of microaggregates [42]. In this study, we measured PDMP and MDMP to assess coagulation in Type II diabetes. Concentrations of plasma PDMP and MDMP were both significantly higher in Type II diabetes patients than in control subjects. PDMPs play an important role in coagulation. A study found that C5b-9 and thrombin increase the number of binding sites for factor V on PDMPs [43]. They also showed that PDMPs play a major role in production of thrombin by the factor Va-Xa complex and that PDMPs are responsible for the procoagulant activity of platelets. Thus, increased concentrations of PDMPs could cause hypercoagulability. Increased PDMP concentrations are

Table 3. Relation between platelet activation markers, microparticles, and sE-selectin in Type II diabetes patients with nephropathy $(n=29)$

\begin{tabular}{lll}
\hline & \multicolumn{2}{l}{ sE-selectin } \\
\cline { 2 - 3 }$r$ & $p$ value \\
\hline MDMP & 0.465 & $p<0.01$ \\
PDMP & 0.422 & $p<0.05$ \\
plt-CD62P & 0.485 & $p<0.01$ \\
plt-CD63 & 0.439 & $p<0.01$ \\
\hline
\end{tabular}


also observed in uremia and cerebral infarction [34]. In addition, increased PDMP levels occur in disease states where activated platelets have been detected in vivo $[12,34]$. Previously, we reported that PDMP concentrations are significantly higher in diabetic patients with higher serum LDL than in similar patients with lowered serum LDL, which suggests that PDMPs participate in the development or progression of atherosclerosis in patients with diabetes mellitus [12]. In this study, we found that the concentration of MDMPs in Type II diabetic patients are higher than those in control subjects. Investigators reported that co-culturing leukocytes with endothelium induces endothelial activation and inflammatory gene induction and that this pathway is mediated by membrane microparticles released from the activated leukocytes [26]. Our current findings suggest that increased PDMPs and MDMPs in Type II diabetic patients could contribute to the development of atherosclerosis.

In this study, no significant differences were detected in binding of anti-GPIIb/IIIa and anti-GPIb monoclonal antibodies between the control and Type II diabetic groups. Concentrations of CD62Pand CD63-positive platelets were higher in Type II diabetic patients than in control subjects. CD62P is glycoprotein located in the a-granule membrane and CD63 is located in the lysosomal integral membrane. When platelets are stimulated, CD62P and CD63 are rapidly redistributed to the platelet surface. This change in CD62P and CD63 localization is considered a marker of platelet activation [31]. Quantitative increases in glycoproteins after platelet activation were not observed in GPIIb/IIIa or GPIb. Flow cytometric analysis of platelet CD62P and CD63 concentrations has been standardized $[31,44]$. With this technique, it has been shown that circulating concentrations of CD62P- and CD63-positive platelets are higher in patients with coronary artery disease and diabetes [44]. Our findings are consistent with those of these previous reports. Our present results on PDMP, MDMP, CD62P, and CD63 concentrations also suggest that enhanced platelet activity and related procoagulant activity in Type II diabetes induce hypercoagulation.

In this study, the activation of monocytes seemed to correlate with the concentration of MDMP in Type II diabetic patients. Concentrations of sE-selectin were also increased significantly in Type II diabetic patients with nephropathy. In general, a subset of monocytes and lymphocytes interact and activate endothelial cells. Increased expression of endothelial adhesion molecules could accelerate vascular damage, and these molecules could be stimulated by oxidized LDL. The above-mentioned adhesion molecules include E-selectin, which is a product of endothelial cells, and P-selectin, a product of endothelial cells and platelets $[45,46]$. In this study, E-selectin in- creased slightly in Type II diabetic patients. Increased activated platelets and PDMPs, which lead to infiltration of monocytes into the arterial wall could be crucial in the development of atherosclerosis [47].

The increased expression of adhesion molecules was associated with activated monocytes. These activated monocytes could bind to vascular endothelial cells, and this binding could contribute to the development of atherosclerosis in Type II diabetic patients. PDMPs and activated platelets could stimulate the activation of monocytes and promote the production of MDMPs. In our study, both MDMPs and PDMPs showed a positive correlation with activated platelets. Because MDMP concentration was especially high in the diabetic patients with nephropathy, we suggest that MDMP concentration in Type II diabetes patients could be a useful indicator of nephropathy progression.

In conclusion, we measured and compared the concentrations of plasma MDMP and platelet activation markers to develop a better understanding of their potential contribution to diabetic vascular complications. Concentrations of CD62P- and CD63-positive platelets, and of plasma PDMP, MDMP, and sEselectin were significantly higher in Type II diabetic patients than in control subjects. Monocyte activation was enhanced and could be the effect of stimulation by activated platelets and PDMPs in Type II diabetic patients. The activation of monocytes could also cause vascular endothelial cell damage. These findings suggest that elevated MDMPs could be a sign of vascular complication in Type II diabetic patients, particularly those who suffer from diabetes-associated nephropathy.

Acknowledgements. This study was partly supported by a grant from the Japan Foundation of Neuropsychiatry and Hematology Research, A Research Grant for Advanced Medical Care from the Ministry of Health and Welfare of Japan, and a Grant (13670760 to S. N.) from the Ministry of Education, Science and Culture of Japan.

\section{References}

1. Turney JH, Woods HF, Fewell MR, Weston MJ (1981) Factor VIII complex in uraemia and effects of haemodialysis. BMJ 282: $1653-1656$

2. Ron D, Oren I, Aviram M, Better OS, Brook JG (1983) Accumulation of lipoprotein remnants in patients with chronic renal failure. Atherosclerosis 46: 67-75

3. Lopes-Virella MF, Virella G (1992) Immune mechanisms of atherosclerosis in diabetes mellitus. Diabetes 41 [Suppl 2]: 86-91

4. Carmassi F, Morale M, Puccetti R et al. (1992) Coagulation and fibrinolytic system impairment in insulin dependent diabetes melliyus. Thromb Res 67: 643-654

5. Steiner G (1985) Atherosclerosis, the major complication of diabetes. Adv Exp Med Biol 189: 277-297

6. Uusitupa MI, Niskanen LK, Siitonen O, Voutilainen E, Pyorala K (1993) Ten years cardiovascular mortality in relation to risk factors and abnormalities in lipoprotein composition in 
Type II (non-insulin-dependent) diabetic and non-diabetic subjects. Diabetologia 36: 1175-1184

7. Baumgartner-Parzer SM, Wagner L, Pettermann M, Gessl A, Waldhausl W (1995) Modulation by high glucose of adhesion molecule expression in cultured endothelial cells. Diabetologia 38: $1367-1370$

8. Lampeter ER, Kishimoto TK, Rothlein R et al. (1992) Elevated levels of circulating adhesion molecules in IDDM patients and in subjects at risk for IDDM. 41: 1668-1671

9. Steiner M, Reinhardt KM, Krammer B, Ernst B, Blann AD (1994) Increased levels of soluble adhesion molecules in type 2 (non-insulin dependent) diabetes mellitus are independent of glycaemic control. Thromb Haemost 72: 979-984

10. Cominacini L, Pasini AF, Garbin U et al. (1995) Elevated levels of soluble E-selectin in patients with IDDM and NIDDM: relation to metabolic control. Diabetologia 38: 1122-1124

11. Cominacini L, Pasini AF, Garbin U et al. (1997) E-selectin plasma concentration is influenced by glycaemic control in NIDDM patients: possible role of oxidative stress. Diabetologia 40: 584-589

12. Nomura S, Suzuki M, Katsura K et al. (1995) Platelet-derived microparticles may influence the development of atherosclerosis in diabetes mellitus. Atherosclerosis 116: 235-240

13. Nomura S, Shouzu A, Omoto S et al. (1998) Effect of cilostazol on soluble adhesion molecules and platelet-derived microparticles in patients with diabetes. Thromb Haemost 80: 388-392

14. Omoto S, Nomura S, Shouzu A et al. (1999) Significance of platelet-derived microparticles and activated platelets in diabetic nephropathy. Nephron 81: 271-277

15. Nomura S, Shouzu A, Omoto S, Nishikawa M, Fukuhara S (2000) Significance of chemokines and activated platelets in patients with diabetes. Clin Exp Immunol 121: 437-443

16. Johnson RB (1988) Monocytes and macrophages. N Engl J Med 318: 747-752

17. Ross R (1993) The pathogenesis of atherosclerosis: a perspective study for the 1990s. Nature 362: 801-809

18. Fruqi RM, DiCorleto PE (1993) Mechanisms of monocyte recruitment and accumulation. Br Heart J 69: S19-S29

19. Beekhuizen H, van Furth R (1993) Monocyte adherence to human vascular endothelium. J Leukoc Biol 54: 363-378

20. Jonasson L, Holm J, Bondjers G, Hansson GK (1986) Regional accumlation of Tcells, macrophages, and smooth muscle cells in the human atherosclerotic plaque. Arteriosclerosis 6: 131-138

21. Drake TA, Ruf W, Morrissey JH, Edginton TS (1989) Functional tissue factor is entirely surface expressed on lipopolysaccharide stimulated human blood monocytes and a constitutively tissue factor producing neoplastic cell line. J Cell Biol 109: 389-394

22. Wilcox JN, Smith KM, Schwartz SM, Gordon D (1989) Localization of tissue factor in normal vessel wall and in the atherosclerotic plaque. Proc Natl Acad Sci USA 86: 2839-2843

23. Furie B, Furie BC (1988) The molecular basis of blood coagulation. Cell 53: 505-518

24. Osnes LTN, Westvik AB, Kieruf P (1994) Procoagulant and profibrinolytic activities of cryopreserved human monocytes. Thromb Res 76: 373-383

25. Satta N, Toti F, Feugeas O et al. (1994) Monocyte vesiculation is a possible mechanism for dissemination of membrane-associated procoagulant activities and adhesion molecules after stimulation by lipopolysaccharide. J Immunol 153: 3245-3255

26. Mesri M, Altieri DC (1998) Endothelial cell activation by leukocyte microparticles. J Immunol 161: 4382-4387

27. Robinson RA, Worfolk L, Tracy PB (1992) Endotoxin enhances the expression of monocyte prothrombinase activity. Blood 79: 406
28. Mallat Z, Hugel B, Ohan J, Leseche G, Freyssinet JM, Tedgui A (1999) Shed membrane microparticles with procoagulant potential in human atherosclerotic plaques. Circulation 99: 348-353

29. Fries, JWU, Williams AJ, Atkins RC, Newman W, Lipscomb MF, Collins T (1993) Expression of VCAM-1 and E-selectin in an in vivo model of endothelial activation. Am J Pathol 143: $725-737$

30. Davies MJ, Gordon JL, Gearing AJH et al. (1993) The expression of the adhesion molecules ICAM-1, VCAM-1, PECAM-1, and E-selectin in human atherosclerosis. J Pathol 171: 223-229

31. Murakami T, Komiyama Y, Masuda M et al. (1996) Flow cytometric analysis of platelet activation markers CD62P and CD63 in patients with coronary artery disease. Eur J Clin Invest 26: 996-1003

32. Miyazaki Y, Nomura S, Miyake Tet al. (1996) High shear stress can initiate both platelet aggregation and shedding of procoagulant containing microparticles. Blood 88: 3456-3464

33. Nomura S, Suzuki M, Kido H et al. (1992) Differences between platelet and microparticle glycoprotein IIb/IIIa. Cytometry 13: 621-629

34. Nomura S, Komiyama Y, Miyake T et al. (1994) Amyloid $\beta$ protein precursor-rich platelet microparticles in thrombotic disease. Thromb Haemost 72: 519-522

35. Miyake T, Nomura S, Komiyama Y et al. (1997) Effect of a new monoclonal anti-glycoprotein IX antibody, KMP-9, on high shear-induced platelet aggregation. Thromb Haemost 78: 902-909

36. Nomura S, Nagata H, Oda K, Kokawa T, Yasunaga K (1987) Effects of EDTA on membrane glycoprotein IIb-IIIa complex: analysis using flow cytometry.Thromb Res 47: 47-58

37. Yanabu M, Ozaki Y, Nomura S et al. (1997) Tyrosine phosphorylation and p72syk activation by an anti-glycoprotein Ib monoclonal antibody. Blood 89: 1590-1598

38. Xie GL, Nomura S, Fukuhara S (1997) Annexin V expression and membrane vesiculation during activation of leukemic cell lines. Haemostasis 27: 259-268

39. Auwerx J, Bouillon R, Collen D, Geboers J (1988) Tissue-type plasminogen activator antigen and plasminogen activator inhibitor in diabetes mellitus. Atherosclerosis 8: 68-72

40. Panahloo A, Mohamed-Ali V, Lane A, Green F, Humphries SE, Yudkin JS (1995) Determinants of plasminogen activator inhibitor 1 activity in treated NIDDM and its relation to a polymorphism in the plasminogen activator inhibitor 1 gene. Diabetes 44: 37-42

41. Kruszynska YT, Yu JG, Olefsky JM, Sobel BE (2000) Effects of troglitazone on blood concentrations of plasminogen activator 1 in patients with type 2 diabetes and in lean and obese normal subjects. Diabetes 49: 633-639

42. Packham MA, Mustard JF (1986) The role of platelets in the development and complications of atherosclerosis. Semin $\mathrm{He}-$ matol 23: 8-19

43. Sims PJ, Faioni EM, Wiedmer T, Shattil SJ (1988) Complement proteins C5b-9 cause release of membrane vesicles from the platelet surface that are enriched in the membrane receptor for coagulation factor $\mathrm{Va}$ and express prothrombinase activity. J Biol Chem 263: 18205-18212

44. Tschope D, Spangenberg P, Esser J et al. (1990) Flow cytometric detection of surface membrane alterations and concomitant changes in the cytoskeletal actins status of activated platelets. Cytometry 11: 652-656

45. Springer TA (1990) Adhesion receptors of the immune system. Nature 346: 425-434

46. Bevilacqua MP (1993) Endothelial-leukocyte adhesion molecules. Annu Rev Immunol 1: 767-804

47. Libby P (1996) Atheroma: more than mush. Lancet 348: S4-S7 